\title{
Model of Short- and Long-Term Outcomes of Emicizumab Prophylaxis Treatment for Persons with Hemophilia A
}

\author{
Zheng-Yi Zhou, PhD; Karina Raimundo, MS; Anisha M. Patel, PhD, MS; Simeng Han, PhD; Yusi Ji, PhD; \\ Honghao Fang, MS; Jia Zhong, PhD; Keith A. Betts, PhD; and Arash Mahajerin, MD
}

\begin{abstract}
BACKGROUND: Hemophilia A (HA) can result in bleeding events because of low or absent clotting factor VIII (FVIII). Prophylactic treatment for severe HA includes replacement FVIII infusions and emicizumab, a bispecific factor IXa- and factor X-directed antibody.
\end{abstract}

OBJECTIVE: To develop an economic model to predict the short- and longterm clinical and economic outcomes of prophylaxis with emicizumab versus short-acting recombinant FVIII among persons with HA in the United States.

METHODS: A Markov model was developed to compare clinical outcomes and costs of emicizumab versus FVIII prophylaxis among persons with severe HA from U.S. payer and societal perspectives. Patients started prophylaxis at age 1 year in the base case. Mutually exclusive health states considered were "no arthropathy," "arthropathy," "surgery," and "death." Serious adverse events, breakthrough bleeds, and inhibitor development were simulated throughout the modeled time horizon. In addition to the prophylaxis drug costs, patients could incur other direct costs related to breakthrough bleeds treatment, serious adverse events, development of inhibitors, arthropathy, and orthopedic surgery. Indirect costs associated with productivity loss (i.e., missed work or disabilities) were applied for adults. Model inputs were obtained from the HAVEN 3 trial, published literature, and expert opinion. The model used a lifetime horizon, and results for 1 year and 5 years were also reported. Deterministic sensitivity analyses and scenario analyses were conducted to assess robustness of the model.

RESULTS: Over a lifetime horizon, the cumulative number of all treated bleeds and joint bleeds avoided on emicizumab versus FVIII prophylaxis were 278.2 and 151.7, respectively. Correspondingly, arthropathy (mean age at onset: 12.9 vs. 5.4 years) and FVIII inhibitor development (mean age at development: 13.9 vs. 1.1 years) were delayed. Total direct and indirect costs were lower for emicizumab versus FVIII prophylaxis for all modeled time horizons $(\$ 97,159$ vs. $\$ 331,610$ at 1 year; $\$ 603,146$ vs. $\$ 1,459,496$ at 5 years; and $\$ 15,238,072$ vs. $\$ 22,820,281$ over a lifetime horizon). The sensitivity analyses indicated that clinical outcomes were sensitive to efficacy inputs, while economic outcomes were driven by the discount rate, dosing schedules, and treatments after inhibitor development. Results for moderate to severe patients were consistent with findings in the severe $\mathrm{HA}$ population.

CONCLUSIONS: The model suggests that emicizumab prophylaxis confers additional clinical benefits, resulting in a lower number of bleeding events and delayed onset of arthropathy and inhibitor development across all time assessment horizons. Compared with short-acting recombinant FVIII, emicizumab prophylaxis leads to superior patient outcomes and cost savings from U.S. payer and societal perspectives.

J Manag Care Spec Pharm. 2020;26(9):1109-20

Copyright $\odot 2020$, Academy of Managed Care Pharmacy. All rights reserved.

\section{What is already known about this subject}

Hemophilia A (HA), a rare X-chromosome-linked genetic disorder, can result in bleeding events because of low or absent levels of clotting factor VIII (FVIII).

Prophylactic treatments for persons with severe HA include replacement infusions of FVIII and emicizumab, a bispecific factor IXa- and factor X-directed antibody.

\section{What this study adds}

This economic model is the first to predict the short- and longterm clinical and economic outcomes of prophylaxis with emicizumab versus recombinant FVIII among persons with HA Compared with FVIII, prophylactic treatment of HA with emicizumab was projected to have superior clinical outcomes and a lower financial impact from U.S. payer and societal perspectives.

工 emophilia A (HA) is a rare X-chromosome-linked bleeding disorder affecting approximately 1 in 5,000 live male births and 20,000 individuals in the United States. ${ }^{1}$ HA ranges from clinically mild to severe and correlates with endogenously produced clotting factor VIII (FVIII) levels. Persons with severe HA can experience frequent spontaneous bleeds into joints or muscles, leading to pain, swelling, arthropathy, and life-threatening bleeds such as intracranial hemorrhage. ${ }^{2,3}$ In addition, bleeding events can result in occupational impairment and progressive mobility loss.

To prevent bleeding episodes, prophylaxis with exogenous FVIII is recommended for persons with severe HA. ${ }^{4-6}$ The optimal form of this treatment requires intravenous administration of FVIII 1-3 times weekly, imposing a substantial treatment burden on patients with severe HA, particularly those with poor venous access. ${ }^{4,5}$ Frequent FVIII administration is also associated with suboptimal treatment adherence, which can negatively affect treatment outcomes. ${ }^{7}$ In addition, therapeutic FVIII is immunogenic and increases the risk of developing antibodies to exogenous FVIII (FVIII inhibitors), particularly within the first 50 exposure days. ${ }^{8-11}$ Persons with HA who have developed FVIII inhibitors are often treated with bypassing agents (BPA) prophylactically and/or for acute bleeds, as well as with FVIII for immune tolerance induction (ITI), ${ }^{12}$ all of which imposes additional economic and treatment burdens. 


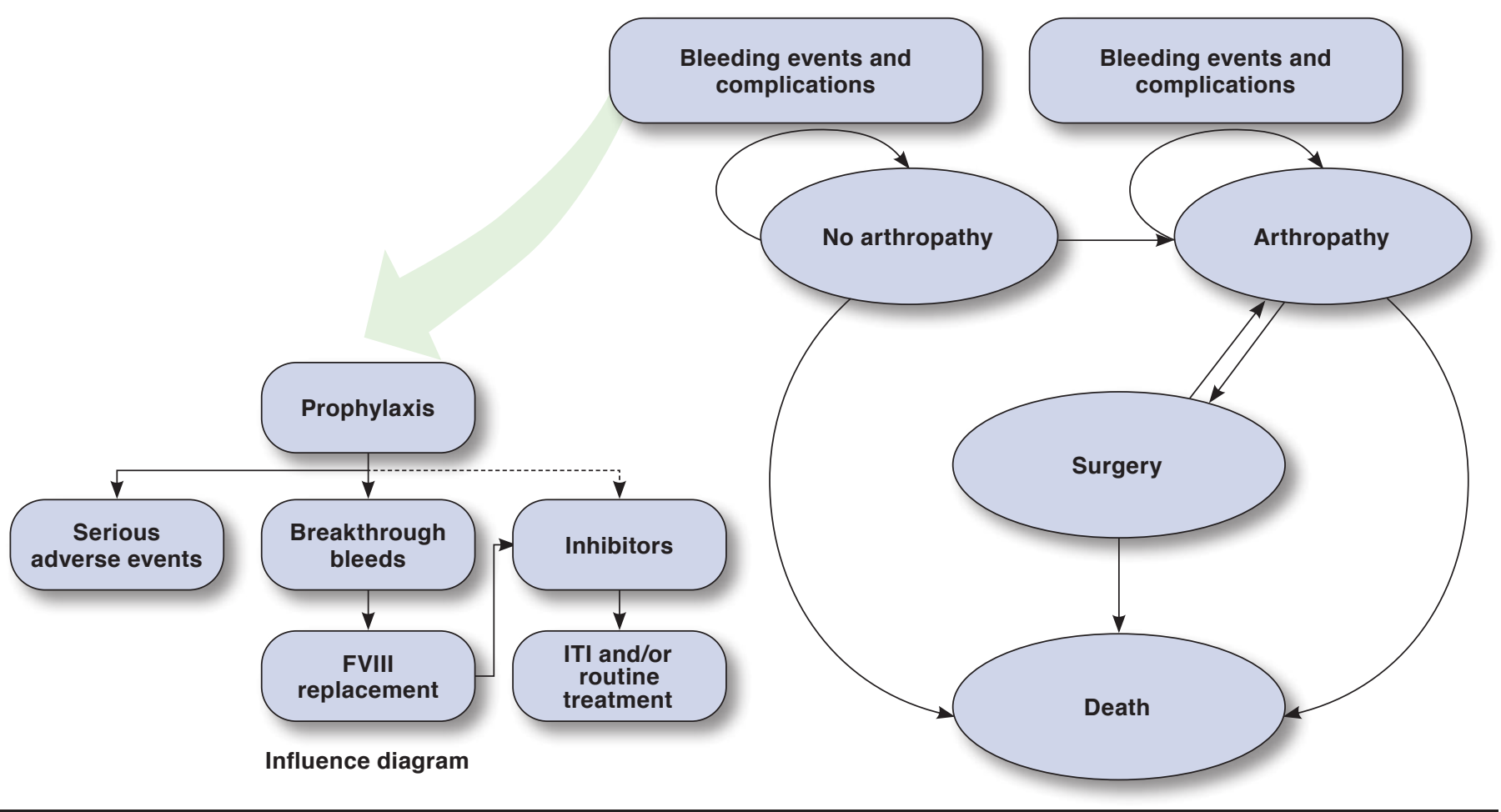

Note: The dashed line indicates inhibitor development directly attributable to receiving prophylaxis, which only occurs in the FVIII arm. FVIII = factor VIII; ITI = immune tolerance induction .

Emicizumab, a humanized bispecific factor IXa- and factor X-directed antibody, is a novel therapy for HA that overcomes some of the shortfalls of FVIII prophylaxis. ${ }^{13}$ It is approved for prophylaxis to prevent or reduce the frequency of bleeding episodes in persons with HA with or without FVIII inhibitors. ${ }^{14}$ Because of its unique chemical structure, emicizumab does not induce or is affected by FVIII inhibitors. ${ }^{13,15}$ The long half-life of emicizumab allows less frequent administration (once every 1 , 2 , or 4 weeks), which reduces the treatment burden..$^{16}$ In addition, the clinical outcomes of persons with HA with and without inhibitors treated with emicizumab prophylaxis are superior to those treated with FVIII prophylaxis. ${ }^{15,17,18}$ The improved control of bleeding with emicizumab, as well as the prevention or deferral of inhibitor development, may decrease HA complications and, consequently, reduce medical costs and productivity loss.

With the entry of a novel prophylactic treatment for HA, comparative studies are needed to provide insight into the clinical and economic value, costs, and benefits compared with standard therapies. Thus, the primary objective of this study was to predict the short- and long-term clinical and economic outcomes of prophylaxis with emicizumab versus FVIII among persons with HA using a modeling approach.

\section{Methods}

\section{Model Overview}

A Markov state transition model was developed in Excel 2016 (Microsoft, Redmond, WA) to predict the short- and long-term clinical and economic outcomes of emicizumab versus shortacting recombinant FVIII prophylaxis among persons with HA in the base case. The model was adapted from Colombo et al. (2011) and consisted of 4 mutually exclusive health states: "no arthropathy," "arthropathy," "surgery," and "death" (Figure 1). ${ }^{19}$ Patients entered the model and stayed in 1 of the 4 health states in a given cycle, with "death" as the absorbing state. Events such as serious adverse events (SAEs), breakthrough bleeds (BTBs), and inhibitor development were simulated at each cycle. Patients were assumed to have had no previous exposure to FVIII, no FVIII inhibitors, and received no lifelong prophylaxis with emicizumab or FVIII unless developing inhibitors. Because of the extreme rarity of females with HA, the base-case population was restricted to male children with severe HA initiating prophylaxis at the age of 1 year without any joint damage..$^{20,21}$

The cohort-level simulation was conducted in weekly cycles over 99 years, representing a lifetime horizon for this population. 


\section{Parameter}

Annual Bleed Rate

Severe without inhibitors ${ }^{\mathrm{a}, 15}$

Severe with inhibitors (aged $<12$ years) $15,17,18$

Severe with inhibitors (aged $\geq 12$ years)

Moderate without inhibitors ${ }^{\mathrm{a}, 15,22}$

Moderate with inhibitors (aged $<12$ years) $15,17,18,22$

Moderate with inhibitors (aged $\geq 12$ years) $15,17,18,22$

Risk of developing inhibitors

Mean exposure duration before developing inhibitors (days) ${ }^{\mathrm{c}}$

Proportion with persistent inhibitors ${ }^{11}$

Treatment of inhibitors

\begin{tabular}{|c|c|c|}
\hline Proportion receiving ITI treatment ${ }^{9}$ & \multicolumn{2}{|c|}{$60 \%$} \\
\hline Overall success rate of ITI treatment 30 & $41.4 \%$ & $38.6 \%$ \\
\hline Mean treatment duration (months) ${ }^{30}$ & 23.7 & 20.5 \\
\hline \multicolumn{3}{|l|}{ Unit drug cost } \\
\hline Wholesale acquisition cost $^{31}$ & $\$ 99.20$ per mg & $\$ 1.58$ per IU \\
\hline \multicolumn{3}{|l|}{ Dosing schedule } \\
\hline \multirow{2}{*}{ Prophylaxis ${ }^{15,29}$} & 3 mg/kg QW (weeks 1-4) & \multirow{2}{*}{30 IU/kg every other day } \\
\hline & $1.5 \mathrm{mg} / \mathrm{kg}$ QW (weeks 5+) & \\
\hline ITI treatment 30 & $50 \mathrm{IU} / \mathrm{kg}$ TIW & $200 \mathrm{IU} / \mathrm{kg} \mathrm{QD}$ \\
\hline FVIII use for BTB 33,34 & \multicolumn{2}{|c|}{$50.4 \mathrm{IU} / \mathrm{kg}$} \\
\hline \multicolumn{3}{|l|}{ Annual treatment cost for PwHA with inhibitors ${ }^{33,34}$} \\
\hline PwHA aged $<12$ years & $\$ 246,645$ & $\$ 708,606$ \\
\hline PwHA aged $\geq 12$ years & $\$ 707,614$ & $\$ 1,791,577$ \\
\hline \multicolumn{3}{|l|}{ Medical cost } \\
\hline No arthropathy (per year) & \multicolumn{2}{|c|}{$\$ 919$} \\
\hline Arthropathy (per year) & \multicolumn{2}{|c|}{$\$ 1,584$} \\
\hline Surgery (one-time) 25,35 & \multicolumn{2}{|c|}{$\$ 55,518$} \\
\hline BTB (per episode) $36-38$ & \multicolumn{2}{|c|}{$\$ 578$} \\
\hline CVAD infection during prophylaxis (one-time) $36,51,52$ & NA & $\$ 4,594$ \\
\hline CVAD infection during ITI treatment (one-time) $36,51,52$ & \multicolumn{2}{|c|}{$\$ 20,508$} \\
\hline \multicolumn{3}{|l|}{ Serious adverse event cost } \\
\hline Serious adverse event cost (one-time) d,36 & $\$ 930$ & $\$ 1,429$ \\
\hline \multicolumn{3}{|l|}{ Indirect cost (per week) 39,40} \\
\hline Pettersson score of $0-4$ & \multicolumn{2}{|c|}{$\$ 137$} \\
\hline Pettersson score of 5-27 & \multicolumn{2}{|c|}{$\$ 502$} \\
\hline Pettersson score of 28-78 & \multicolumn{2}{|c|}{$\$ 1,160$} \\
\hline
\end{tabular}

Note: All costs were inflated to 2018 U.S. dollars.

aThe ABRs for FVIII prophylaxis were obtained from Genentech (data on file, noninterventional study [protocol BH29768]).

${ }^{b}$ The ABRs of all treated bleeds were based on the average rates of a treatment mix of prophylaxis with emicizumab or bypassing agents.

'Input from expert clinical opinion.

dThe SAE rates for FVIII prophylaxis were obtained from Genentech (data on file, noninterventional study [protocol BH29768]).

$A B R=$ annual bleed rate; $B T B=$ break-through bleed; $C V A D=$ central venous access device; FVIII=factor VIII; HA=hemophilia A; ITI=immune tolerance induction;

NA = not applicable; PwHA= persons with hemophilia $A ;$ QD = once a day; QW=once a week; SAE=serious adverse event; TIW=3 times a week.

The clinical and economic outcomes for emicizumab versus FVIII prophylaxis were compared over a lifetime horizon, as well as at 1 and 5 years. The model was conducted from U.S. third-party payer and societal perspectives, considering direct and indirect costs. Effectiveness and costs were discounted at an annual rate of 3\%. A table summarizing key model inputs and assumptions is displayed in Appendix A (available in online article)
Because this model only used previously published data, no institutional board review was required.

\section{Model Inputs}

Efficacy. Efficacy inputs used in the model are summarized in Table 1. Model inputs were collected from the HAVEN 1, HAVEN 2, and HAVEN 3 trials or through a targeted literature search. ${ }^{15,17,18}$ HAVEN 1, HAVEN 2, and HAVEN 3 were all 
phase 3 trials of emicizumab prophylaxis, yet with different study populations (aged $\geq 12$ years with inhibitors, aged $<12$ years with inhibitors, and aged $\geq 12$ years without inhibitors, respectively). Clinical expert opinion was used in the absence of supporting data.

The annual bleed rates (ABRs) from HAVEN $3^{15}$ and a noninterventional study (NIS; data on file, Genentech, noninterventional study [protocol BH29768]) were used for persons with severe HA before developing inhibitors. After developing inhibitors, the ABRs of all treated bleeds were based on the average rates of a treatment mix of prophylaxis with emicizumab or BPA, ${ }^{17,18}$ and the ABRs of treated joint bleeds were estimated with a rate ratio from HAVEN $3 .{ }^{15}$ For moderate HA, a rate ratio (0.667 for moderate vs. severe) was derived from Berntorp et al. (2017) and applied to ABRs for persons with severe HA. ${ }^{22}$

Development of Inhibitors. FVIII inhibitors may develop contingent on the cumulative exposure to FVIII, assumed to be 20 days based on clinical expert opinion. Among patients with inhibitors, $27.1 \%$ would have persistent inhibitors and require treatment. ${ }^{11}$ Additionally, $60 \%$ of patients with persistent inhibitors were assumed to have high-titer ( $>5$ Bethesda units $/ \mathrm{mL}$ ) inhibitors and receive ITI. ${ }^{9}$ Prophylactic treatments with emicizumab or BPA would be administered to the remaining patients with low-titer $(\leq 5$ Bethesda units/mL) persistent inhibitors and those who failed ITI. It was assumed that 100\% of patients in the emicizumab arm continued receiving emicizumab after developing inhibitors, while $50 \%$ of patients in the FVIII arm went on to receive emicizumab, and the other 50\% received BPA.

Joint Damage. Arthropathy and surgery were estimated based on the ABR of treated joint bleeds, converted to a weekly probability of a joint bleed and was measured by the Pettersson score. ${ }^{23}$ The Pettersson score starts at 0 and increases by 1 with every 12.6 joint bleeds. ${ }^{24}$ A Pettersson score of 0 indicates no arthropathy, while a score of $\geq 1$ indicates arthropathy, and orthopedic surgery would be triggered at a score of $28 .{ }^{25}$ Only patients aged $<80$ years were eligible for joint surgery. ${ }^{25}$ All joint surgeries were considered to be successful. After each surgery, patients were assumed to return to the "arthropathy" state with minor joint damage (Pettersson score was assumed to be 1$)$.

Mortality. The goal of prophylaxis is to maintain the blood clotting factor level close to that of patients with mild to moderate HA; therefore, patients were assumed to have the same risk of death as patients with mild to moderate HA. The risk was estimated by applying a rate ratio of 1.2 to the age-specific natural mortality rates for males from the U.S. life tables..$^{26-28}$ Respective mortality rates for treatment with emicizumab versus FVIII are not available, so they were assumed to be the same.
Costs. Costs, including treatment costs, medical costs, SAE costs, and indirect costs were estimated based on the literature or public data (Table 1). All costs were inflated to 2018 U.S. dollars.

Treatment Costs. Patients incurred treatment costs associated with prophylaxis. In addition, patients could incur other treatment costs related to BTB and treatment for FVIII inhibitors including ITI. Wholesale acquisition cost (WAC) was used as the base-case unit price. WAC for Advate (antihemophilic factor [recombinant]) was used to estimate costs for FVIII, given its large U.S. market share. Dosing schedules for prophylaxis were based on HAVEN 3 and the U.S. Food and Drug Administration prescribing information. ${ }^{15,29}$ The mean FVIII use for each BTB was estimated based on HAVEN 3.

For patients receiving ITI, dosing schedules, mean treatment durations, and overall success rates of high- and lowdose ITI were based on Hay et al. (2012)..$^{30}$ The annual costs for postinhibitor routine treatments were calculated using the WAC unit price, average body weight, and dose/bleed data from HAVEN 1 and HAVEN 2.17,18,31,32

Medical Costs. Costs associated with arthropathy were calculated annually based on the frequencies of resource use reported in O'Hara et al. (2018), ${ }^{33}$ and the unit costs published in the Centers for Medicare \& Medicaid Services Physician Fee Schedule. ${ }^{34}$ Surgery costs included costs for orthopedic surgery and postsurgery recovery. ${ }^{25,35}$ Patients were assumed to stay in the "surgery" state for 1 cycle and then move back to the "arthropathy" state (Pettersson score reset to 1 after surgery for the base case). Costs associated with a central venous access device (CVAD) infection were considered as 1-time costs when patients received FVIII prophylaxis or ITI treatment. The unit cost of managing a CVAD infection was based on values published by the Healthcare Cost and Utilization Project Network (HCUPnet). ${ }^{36}$ Hospitalization and emergency room visits were also considered when BTB occurred, with the unit costs based on HCUPnet and Zakieh et al. (2017), respectively. ${ }^{36,37}$ The frequency of resource use per BTB episode was based on Zhou et al. (2015). ${ }^{38}$

SAE Costs. SAEs, which can be life threatening and generally require or prolong hospitalization, were considered. Rates of SAE were based on HAVEN $3^{15}$ and the NIS (data on file, Genentech, noninterventional study [protocol BH29768]). For patients in the emicizumab prophylaxis arm, pooled data from Arm A (1.5 mg/kg once a week) and Arm B (3 mg/kg once every 2 weeks) in HAVEN 3 were used. Costs for SAEs were 1-time costs applied at the beginning of the model. The unit cost of each SAE was derived from HCUPnet. ${ }^{36}$

Indirect Costs. Only indirect costs associated with productivity loss due to part-time work and unemployment were considered. Other indirect costs (e.g., caregiver's unpaid time) were not considered. Weekly missed working hours were measured 
TABLE 2 Base-Case Results: Clinical Outcomes over 1 Year, 5 Years, and the Lifetime of Previously Untreated Persons Aged 1 Year with Severe Hemophilia A Receiving Emicizumab or FVIII Prophylaxis

\begin{tabular}{|c|c|c|c|c|c|c|c|c|c|}
\hline & \multicolumn{3}{|c|}{1 Year } & \multicolumn{3}{|c|}{5 Years } & \multicolumn{3}{|c|}{ Lifetime } \\
\hline & $\begin{array}{l}\text { Emicizumab } \\
\text { Prophylaxis }\end{array}$ & $\begin{array}{c}\text { FVIII } \\
\text { Prophylaxis }\end{array}$ & $\begin{array}{c}\text { Difference } \\
\text { (Emicizumab } \\
\text { vs. FVIII) }\end{array}$ & $\begin{array}{l}\text { Emicizumab } \\
\text { Prophylaxis }\end{array}$ & $\begin{array}{c}\text { FVIII } \\
\text { Prophylaxis }\end{array}$ & $\begin{array}{c}\text { Difference } \\
\text { (Emicizumab } \\
\text { vs. FVIII) }\end{array}$ & $\begin{array}{l}\text { Emicizumab } \\
\text { Prophylaxis }\end{array}$ & $\begin{array}{c}\text { FVIII } \\
\text { Prophylaxis }\end{array}$ & $\begin{array}{c}\text { Difference } \\
\text { (Emicizumab } \\
\text { vs. FVIII) }\end{array}$ \\
\hline \multicolumn{10}{|l|}{ Clinical outcomes } \\
\hline $\begin{array}{l}\text { Cumulative number } \\
\text { of bleeds }\end{array}$ & 1.5 & 5.2 & $\begin{array}{l}3.8 \text { bleeds } \\
\text { avoided }\end{array}$ & 7.4 & 26.3 & $\begin{array}{l}18.9 \text { bleeds } \\
\text { avoided }\end{array}$ & 125.7 & 403.9 & $\begin{array}{c}278.2 \text { bleeds } \\
\text { avoided }\end{array}$ \\
\hline $\begin{array}{l}\text { Cumulative number } \\
\text { of joint bleeds }\end{array}$ & 1.1 & 3.2 & $\begin{array}{l}2.1 \text { bleeds } \\
\text { avoided }\end{array}$ & 5.4 & 15.8 & $\begin{array}{l}10.3 \text { bleeds } \\
\text { avoided }\end{array}$ & 92.2 & 243.8 & $\begin{array}{l}151.7 \text { bleeds } \\
\text { avoided }\end{array}$ \\
\hline $\begin{array}{l}\text { Mean age at inhibitor } \\
\text { development (years) }\end{array}$ & 0.9 & 0.1 & $\begin{array}{l}0.8 \text { years } \\
\text { delayed }\end{array}$ & 4.5 & 0.1 & $\begin{array}{l}4.4 \text { years } \\
\text { delayed }\end{array}$ & 13.9 & 1.1 & $\begin{array}{c}12.8 \text { years } \\
\text { delayed }\end{array}$ \\
\hline $\begin{array}{l}\text { Mean age at } \\
\text { arthropathy onset } \\
\text { (years) }\end{array}$ & 0.9 & 0.9 & 0 years & 4.5 & 3.5 & $\begin{array}{l}1 \text { year } \\
\text { delayed }\end{array}$ & 12.9 & 5.5 & $\begin{array}{l}7.4 \text { years } \\
\text { delayed }\end{array}$ \\
\hline
\end{tabular}

Note: All costs were inflated to 2018 U.S. dollars.

FVIII $=$ factor VIII

and reported by Pettersson score. ${ }^{39}$ Weekly productivity loss by Pettersson score was calculated based on the total missed working hours and the average hourly compensation in the United States. ${ }^{40}$

\section{Model Outputs}

The results of the model were reported over a lifetime horizon, as well as at 1 year and 5 years. Clinical outcomes included the predicted cumulative number of all treated bleeds and treated joint bleeds and the mean ages at FVIII inhibitor development and arthropathy onset. Economic outcomes included direct and indirect costs.

Deterministic sensitivity analyses (DSA) were conducted to examine the robustness of the findings and the sensitivity to changing 1 key model input or assumption while holding others constant. Model input values varied by $\pm 25 \%$ (efficacy and costs, except for SAE costs); SAE costs were set at zero or doubled; or values were based on 95\% confidence intervals (CIs) or other literature. In addition, scenario analyses were performed and included (a) using persons with moderate to severe HA as the target population; (b) using average sales price (ASP) costs for emicizumab and FVIII; (c) using the dosing schedule and unit cost of a long-acting FVIII (Eloctate [antihemophilic factor (recombinant), Fc fusion protein]) as representative for recombinant FVIII; (d) assuming patients in the emicizumab arm would never require ITI; (e) assuming patients receiving ITI would always receive high-dose ITI; (f) using data from Su et al. $(2016 ; 31.2 \%)$ as the proportion of patients with persistent inhibitors receiving $\mathrm{ITI}^{41}$; and (g) assuming $100 \%$ of patients on FVIII prophylaxis would receive emicizumab prophylaxis as the postinhibitor routine treatment.

\section{Results}

\section{Base-Case Results}

Clinical Outcomes. The predicted numbers of treated bleeds and treated joint bleeds were consistently lower among persons with severe HA receiving emicizumab versus FVIII prophylaxis across all time horizons. During the first year, patients on emicizumab prophylaxis compared with those on FVIII would experience 1.5 versus 5.2 treated bleeds and 1.1 versus 3.2 treated joint bleeds, respectively. Over 5 years, the estimates rise to 7.4 versus 26.3 treated bleeds and 5.4 versus 15.8 treated joint bleeds, respectively (Table 2). The results over a lifetime horizon are listed in Table 2. Emicizumab prophylaxis avoided a total of 278.2 bleeds and 151.7 joint bleeds in a lifetime that might have warranted treatments compared with FVIII prophylaxis.

Consequently, emicizumab prophylaxis was associated with later onset of arthropathy (mean age $=12.9$ years) compared with FVIII prophylaxis (5.5 years). Thus, the mean age at arthropathy onset was postponed by 7.4 years with emicizumab prophylaxis. Time to inhibitor development was also postponed by 12.8 years among those receiving emicizumab prophylaxis, with the mean age at inhibitor development predicted to be 13.9 years for emicizumab prophylaxis and 1.1 years for FVIII prophylaxis (Table 2).

Economic Outcomes. The total direct costs were lower for emicizumab versus FVIII prophylaxis over different time horizons. The respective costs estimated for emicizumab versus FVIII prophylaxis were $\$ 97,159$ versus $\$ 331,610$ at 1 year; $\$ 603,146$ versus $1,459,496$ at 5 years; and $\$ 15,238,072$ versus $\$ 22,820,281$ over a lifetime horizon. Similarly, indirect costs over a lifetime horizon were lower for emicizumab $(\$ 138,436)$ compared with FVIII prophylaxis $(\$ 312,204)$. Specific cost components of the lifetime horizon are detailed in Figure 2, with treatment costs for both arms (98.6\% and $98.0 \%$ of 

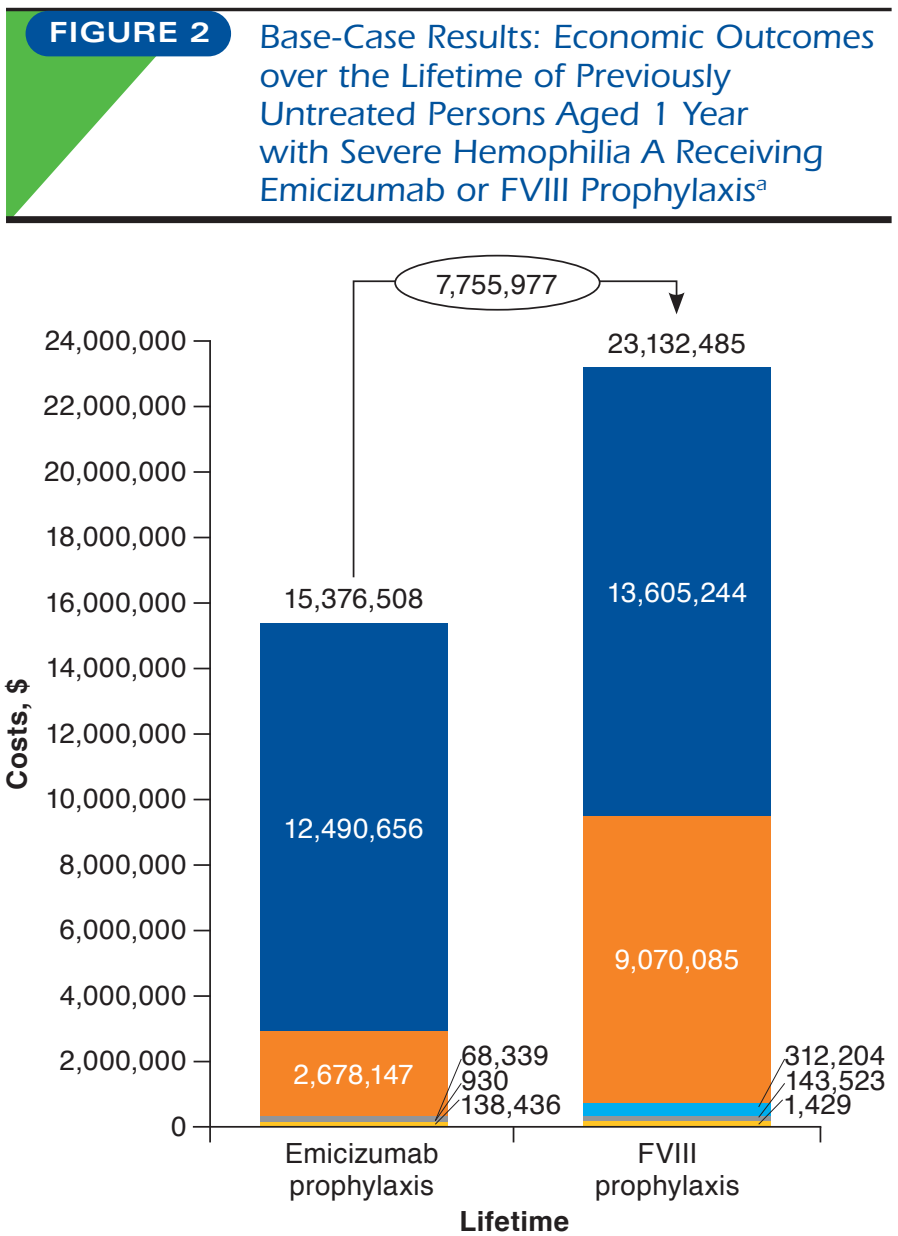

Prophylactic treatment cost
Inhibitor-related treatment cost
Medical cost
Serious adverse event cost
Indirect cost

Note: All costs were inflated to 2018 U.S. dollars.

aThe categories contributing a smaller amount to the total costs included medical costs (\$68,339 for emicizumab and \$143,523 for FVIII); serious adverse event costs (\$930 and \$1,429, respectively); and indirect costs (\$138,436 and \$312,204). FVIII = factor VIII.

the total costs for emicizumab and FVIII prophylaxis over a lifetime horizon, respectively) being the key model driver of overall costs. Cost components over 1- and 5-year horizons are displayed in Appendix B (available in online article).

\section{Deterministic Sensitivity Analysis}

Clinical Outcomes. Among the inputs and scenarios tested, the cumulative number of all treated bleeds avoided over a lifetime ranged from 184.8 to 402.8 , and the cumulative number of treated joint bleeds avoided ranged from 83.2 to
233.9. The DSA results showed that despite variations in model input values, emicizumab prophylaxis decreased the number of treated bleeds, demonstrating the robustness of base-case results. The result was most sensitive to the variation in ABR for patients without inhibitors receiving FVIII prophylaxis, with the number of treated bleeds ranging from 190.0 to 402.8 .

In the scenario using persons with moderate to severe HA as the target population, 257.5 treated bleeds were avoided with emicizumab compared with FVIII prophylaxis. In the scenario where all patients on FVIII received emicizumab as the postinhibitor routine treatment, emicizumab prophylaxis remained a superior option, although the estimated number of avoided treated bleeds fell to 184.8. Other scenarios had a limited effect on number of avoided treated bleeds, with changes of $<3 \%$ from the base case.

Economic Outcomes. Among all inputs and scenarios tested, emicizumab prophylaxis saved $\$ 2,388,783$ to $\$ 21,411,060$ compared with FVIII prophylaxis (Figure 3). The model results were most sensitive to the discount rate, FVIII dosing schedule, and treatments after inhibitor development. Over a lifetime horizon, emicizumab prophylaxis remained a cost-saving option, and the results were robust to variations in model inputs and scenarios.

The lifetime incremental costs among persons with moderate to severe HA were estimated to be $\$ 7,734,325$ lower when using emicizumab versus FVIII prophylaxis, which was similar to the cost savings observed in the severe HA population. The use of long-acting FVIII instead of short-acting FVIII resulted in additional cost savings $(\$ 9,695,476)$. When the unit drug costs of emicizumab and FVIII were based on the ASP, patients in both arms receiving high-dose ITI and all patients on FVIII switching to emicizumab as the postinhibitor routine treatment would have reduced cost savings over a lifetime (25.93\%, $15.16 \%$, and $69.20 \%$ less cost savings, respectively). No other scenarios were found to change economic outcomes more than $10 \%$ from the base case.

\section{Discussion}

HA is a serious and often debilitating disorder; however, prophylactic treatments can prevent or reduce the rate of bleeding. The present economic model is, to our knowledge, the first study to investigate the long-term clinical and economic outcomes of prophylaxis with emicizumab versus FVIII in persons with HA starting treatment without inhibitors. The model's results indicated that emicizumab prophylaxis is expected to lead to superior clinical outcomes compared with FVIII, including reduced bleed rates, postponed onset of arthropathy, and delayed inhibitor development. In addition to better efficacy, emicizumab prophylaxis was associated with lower total costs from U.S. payer and societal perspectives. The lower costs and better effectiveness of emicizumab prophylaxis are 


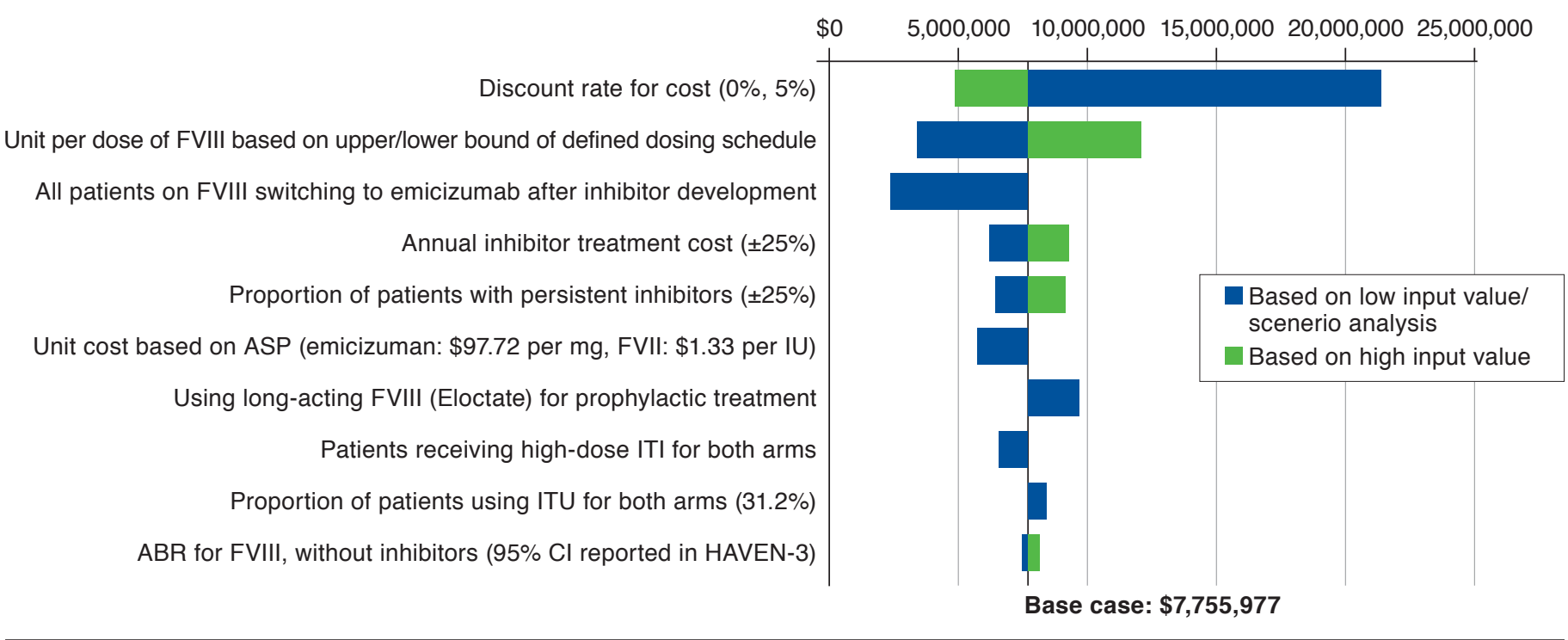

$A B R=$ annual bleed rate; $A S P=$ average sales cost; $C I=$ confidence interval; FVIII = factor VIII; ITI=immune tolerance induction; $I U=$ international unit.

directionally similar to the Institute for Clinical and Economic Review's (ICER) report of emicizumab for the treatment of HA with inhibitors, which showed emicizumab to be dominant over no prophylaxis and prophylaxis with BPA, although with different treatment options following inhibitor development and a different magnitude of impact. ${ }^{42}$

The current study benefits from several strengths, including leveraging trial data to obtain robust clinical inputs. In addition, to depict a complete disease profile over a lifetime horizon, the short-term clinical outcomes of joint bleeds were linked to Pettersson scores to predict the long-term outcomes, including arthropathy and productivity loss. ${ }^{23,39}$ Furthermore, the incorporation of indirect costs added evidence to the effect on productivity loss attributable to hemophilia over a patient's lifetime.

The main model's comparisons were performed in persons with severe HA, while persons with moderate to severe HA were included in the DSA. Under this scenario, emicizumab prophylaxis still had superior clinical and economic benefits and demonstrated that the base-case results were robust to model input variations and assumptions. Thus, the results of this analysis provide valuable evidence to bridge the knowledge gap regarding the economic and clinical values of prophylaxis options for HA and may aid the development of cost-effective and less burdensome treatment options.

In the main model, emicizumab prophylaxis resulted in a reduction in bleeding events compared with FVIII prophylaxis. Specifically, the number of all treated bleeds decreased by $72 \%$ over 1- and 5 -year periods and by $75 \%$ over a lifetime, similar to the results of emicizumab's clinical trials. At the 24-week follow-up in HAVEN 3, emicizumab patients without inhibitors treated had a $68 \%$ decrease in ABR of all treated bleeds compared with FVIII prophylaxis patients. ${ }^{15}$ Likewise, in the short and long term, emicizumab prophylaxis patients experienced approximately one third of the number of treated joint bleeds compared with FVIII prophylaxis patients, similar to the HAVEN trials. ${ }^{15,17,18}$

The current model also predicted that emicizumab prophylaxis would postpone the onset of arthropathy by 7.4 years compared with FVIII prophylaxis, similar to previous findings, given that recurrent joint bleeds lead to arthropathy. ${ }^{43}$ This delay represents a major benefit of emicizumab, given the severe and chronic effect of arthropathy on a person's life. ${ }^{44,45}$ It is also a conservative assumption, since joint arthropathy is multifactorial. Because of improved bleed protection with emicizumab, patients may engage in a more physically active lifestyle that results in better joint health, and the results of HAVEN 2 suggested less bleeding among patients with less joint damage. ${ }^{18}$ However, these benefits are not captured in the current model because of lack of data for patients aged younger than 12 years.

Finally, the model predicted a delay in FVIII inhibitors development by 12.8 years when using emicizumab versus FVIII prophylaxis, likely because emicizumab does not induce or enhance the development of FVIII inhibitors. Based on the assumption of same risk of inhibitor development for emicizumab and FVIII prophylaxis, the model predicted inhibitor 
development at approximately age 14 years for emicizumab. The model then accounted for higher ITI costs incurred in the emicizumab arm (due to higher body weight), but the lifetime results were still cost saving.

In this study, patients were assumed to adhere to the dosing schedules of either therapy throughout their lifetime and not to discontinue treatments. Suboptimal treatment adherence has been identified as an issue in FVIII prophylaxis. ${ }^{7,46}$ Emicizumab prophylaxis offers a more convenient subcutaneous administration and less frequent administration, ${ }^{16}$ which may promote better treatment adherence. However, the effect of suboptimal treatment adherence on clinical outcomes was not captured in the model due to lack of data. Because patients receiving less frequent regimens may be more adherent, not considering treatment adherence can be regarded as a conservative assumption in evaluating the clinical benefits of emicizumab versus FVIII prophylaxis.

The clinical benefits of emicizumab prophylaxis led to further economic benefits in comparison with FVIII. Based on the model, emicizumab prophylaxis was associated with an annual savings of $\$ 234,451$ at 1 year versus FVIII prophylaxis, and the cost savings were sustained long term. Inhibitor-related treatments, including ITI and postinhibitor treatments, comprised $82.4 \%$ of the cost difference. The difference in ITI treatment costs was primarily driven by the dosing schedules.

Because of limited evidence, patients receiving emicizumab and FVIII prophylaxis were assigned to low- and high-dose ITI, respectively, based on clinical expert opinion. The Atlanta Protocol provides another ITI dosing schedule for patients receiving emicizumab. ${ }^{47}$ However, the Atlanta Protocol report was not available until recently, and the data are still preliminary with only 7 patients studied so were not used as clinical inputs in this model. ${ }^{47}$ Per the model, patients could receive emicizumab as a postinhibitor routine treatment, which helped reduce postinhibitor treatment costs because BPA treatments were costlier.

In this analysis, the respective total costs estimated for emicizumab versus FVIII prophylaxis were $\$ 97,159$ versus $\$ 331,610$ at 1 year; $\$ 603,146$ versus $1,459,496$ at 5 years; and $\$ 15,238,072$ versus $\$ 22,820,281$ over a lifetime. The overall assessment of the lower costs and better effectiveness of emicizumab versus FVIII prophylaxis is consistent with a Markov model conducted by ICER (although its population and inputs differed). ${ }^{42}$ A real-world U.S. study reported that the annual costs per person with severe HA treated with FVIII prophylaxis totaled $\$ 301,392$ in $2011,{ }^{38}$ although it did not consider inhibitor treatment costs.

As a disease requiring lifelong treatment, HA is likely to affect individuals' productivity throughout their working years. A study conducted by Fischer et al. (2005) linked indirect costs to Pettersson scores, which accounted for the number of preceding joint bleeds, although this may be a conservative assumption compared with real-world clinical practice. ${ }^{39}$ Thus, the consideration of indirect costs in this study likely increased the comprehensiveness of estimation of the societal economic impact. Specifically, the present model predicted that emicizumab prophylaxis was associated with lower productivity loss compared with FVIII prophylaxis, with indirect costs of $\$ 138,436$ and $\$ 312,204$, respectively.

\section{Limitations}

This study should be interpreted in consideration of its limitations, some of which are inherent to economic modeling studies. First, this model compared prophylaxis treatments only, although some patients are primarily treated on demand (e.g., only in the event of a bleed). However, this study is aligned with the Medical and Scientific Advisory Council recommendations that children with severe HA should receive prophylaxis. ${ }^{48}$

Second, the ABRs for patients without inhibitors were obtained from HAVEN 3, but this trial was conducted on patients without inhibitors aged $\geq 12$ years. A small trial from Japan (HOHOEMI) of pediatric patients without inhibitors found similarly low ABRs when treated with emicizumab. ${ }^{49}$ The HAVEN 1 and HAVEN 2 results suggested that early treatment with emicizumab prophylaxis could greatly decrease the ABR of treated bleeds in patients with inhibitors (0.2 vs. 2.9 in patients aged $<12$ and $\geq 12$ years, respectively). ${ }^{17,18}$ Therefore, applying the ABRs from HAVEN 3 to patients aged $<12$ years is likely to underestimate emicizumab's clinical performance. To address this limitation, a range of ABRs for patients without inhibitors was tested but the conclusions remained unchanged.

Third, long-term ABR data were not available and were assumed the same as within the 24-week trial time frame. The DSA results showed that variations in clinical inputs did not change the conclusions. Pooled data from HAVEN has shown that patients receiving emicizumab maintained a low bleed rate over 120 weeks, ${ }^{50}$ but long-term observations of real-world clinical outcomes are needed to validate the model results.

Fourth, this study did not consider productivity loss of caregivers due to data unavailability, although future analyses might be conducted to account for it.

Finally, this model was not able to account for indirect or intangible costs associated with the psychological effect of living with bleeds and the effect on lifestyle, career, and other choices caused by HA. ${ }^{42}$ Future studies analyzing such aspects of the indirect or intangible costs and effects of HA are suggested.

\section{Conclusions}

This study indicated that, compared with FVIII prophylaxis, prophylactic treatment of HA with emicizumab led to superior clinical outcomes, including a lower number of bleeding events and delayed onset of arthropathy and inhibitor development. In addition, prophylaxis with emicizumab had a substantially lower financial impact from U.S. payer and societal perspectives 
compared with FVIII prophylaxis. Thus, the results of this study highlight the dominance of prophylaxis with emicizumab over FVIII across all time assessment horizons, which may also reduce the burden on patients with HA receiving lifelong treatment.

\section{Authors}

ZHENG-YI ZHOU, PhD, Analysis Group, London, United Kingdom. KARINA RAIMUNDO, MS, and ANISHA M. PATEL, PhD, MS, Genentech, South San Francisco, California. SIMENG HAN, PhD; YUSI JI, PhD; HONGHAO FANG, MS; and JIA ZHONG, PhD, Analysis Group, Beijing, China. KEITH A. BETTS, PhD, Analysis Group, Los Angeles, California, and ARASH MAHAJERIN, MD, CHOC Children's Hospital, Orange, California.

AUTHOR CORRESPONDENCE: Zheng-Yi Zhou, PhD, Analysis Group, 1 Angel Ct., 14th Fl., London, England EC2R 7HJ, UK. Tel.: +44.203.480.7922; Email: Jenny.Zhou@analysisgroup.com.

\section{DISCLOSURES}

Funding for this study was provided by Genentech. Raimundo and Patel are employees of Genentech and own stock or stock options. Zhou, Han, Ji, Fang, Zhong, and Betts are employees of Analysis Group, which received consultancy fees from Genentech for conducting this study. Mahajerin received consultancy fees from Genentech for work on this study.

Portions of this research were presented as a poster at the 2018 American Society of Hematology Conference; December 1-4, 2018; San Diego, CA.

\section{ACKNOWLEDGMENTS}

Medical writing assistance was provided by Shelley Batts, $\mathrm{PhD}$, an employee of Analysis Group.

\section{REFERENCES}

1. Centers for Disease Control and Prevention. Data \& statistics on hemophilia. Available at: https://www.cdc.gov/ncbddd/hemophilia/data.html. Accessed May 11, 2020.

2. Riedl J, Ay C, Pabinger I. Platelets and hemophilia: a review of the literature. Thromb Res. 2017;155:131-39.

3. Manco-Johnson MJ, Soucie JM, Gill JC; Joint Outcomes Committee of the Universal Data Collection- U. S. Hemophilia Treatment Center Network. Prophylaxis usage, bleeding rates, and joint outcomes of hemophilia, 1999 to 2010: a surveillance project. Blood. 2017;129(17):2368-74.

4. Srivastava A, Brewer AK, Mauser-Bunschoten EP, et al. Guidelines for the management of hemophilia. Haemophilia. 2013;19(1):el-47.

5. Ljung R, Gretenkort Andersson N. The current status of prophylactic replacement therapy in children and adults with haemophilia. Br J Haematol. 2015;169(6):777-86

6. Petrini P, Valentino LA, Gringeri A, Re WM, Ewenstein B. Individualizing prophylaxis in hemophilia: a review. Expert Rev Hematol. 2015;8(2):237-46.

7. du Treil S, Rice J, Leissinger CA. Quantifying adherence to treatment and its relationship to quality of life in a well-characterized haemophilia population. Haemophilia. 2007;13(5):493-501.
8. Varthaman A, Lacroix-Desmazes S. Pathogenic immune response to therapeutic factor VIII: exacerbated response or failed induction of tolerance? Haematologica. 2019;104(2):236-44.

9. Witmer C, Young G. Factor VIII inhibitors in hemophilia A: rationale and latest evidence. Ther Adv Hematol. 2013;4(1):59-72.

10. Peyvandi F, Cannavo A, Garagiola I, Palla R, Mannucci PM, Rosendaal FR. Timing and severity of inhibitor development in recombinant versus plasma-derived factor VIII concentrates: a SIPPET analysis. J Thromb Haemost. 2018;16(1):39-43

11. Peyvandi F, Mannucci PM, Garagiola I, et al. A randomized trial of factor VIII and neutralizing antibodies in hemophilia A. N Engl J Med. 2016;374(21):2054-64.

12. Kreuz W, Ettingshausen CE. Inhibitors in patients with haemophilia A. Thromb Res. 2014;134(Suppl 1):S22-26.

13. Shima M, Hanabusa H, Taki M, et al. Factor VIII-mimetic function of humanized bispecific antibody in hemophilia A. N Engl J Med. 2016;374(21):2044-53.

14. Hemlibra (emicizumab-kxwh) injection, for subcutaneous use. Genentech. October 2018. Available at: https://www.accessdata.fda.gov/drugsatfda_docs/ label/2018/761083s002s004lbl.pdf. Accessed May 11, 2020.

15. Mahlangu J, Oldenburg J, Paz-Priel I, et al. Emicizumab prophylaxis in patients who have hemophilia A without inhibitors. N Engl J Med. 2018;379(9):811-22

16. Uchida N, Sambe T, Yoneyama K, et al. A first-in-human phase 1 study of ACE910, a novel factor VIII-mimetic bispecific antibody, in healthy subjects. Blood. 2016;127(13):1633-41

17. Oldenburg J, Mahlangu JN, Kim B, et al. Emicizumab prophylaxis in hemophilia A with inhibitors. N Engl J Med. 2017;377(9):809-18.

18. Young G, Sidonio RF, Liesner R, et al. HAVEN 2 updated analysis: multicenter, open-label, phase 3 study to evaluate efficacy, safety and pharmacokinetics of subcutaneous administration of emicizumab prophylaxis in pediatric patients with hemophilia A with inhibitors. Blood. 2017;130(Suppl 1):85.

19. Colombo GL, Di Matteo S, Mancuso ME, Santagostino E. Cost-utility analysis of prophylaxis versus treatment on demand in severe hemophilia A. Clinicoecon Outcomes Res. 2011;3:55-61.

20. U.S. National Library of Medicine. Hemophilia: inheritance pattern. 2020. Available at: https://ghr.nlm.nih.gov/condition/hemophilia\# inheritance. Accessed May 11, 2020.

21. Centers for Disease Control and Prevention. What is hemophilia? 2020 Available at: https://www.cdc.gov/ncbddd/hemophilia/facts.html. Accessed May 11, 2020.

22. Berntorp E, Dolan G, Hay C, et al. European retrospective study of reallife haemophilia treatment. Haemophilia. 2017;23(1):105-14.

23. Pettersson H, Ahlberg A, Nilsson IM. A radiologic classification of hemophilic arthropathy. Clin Orthop Relat Res. 1980(149):153-59.

24. Fischer K, van Hout BA, van der Bom JG, Grobbee DE, van den Berg HM. Association between joint bleeds and Pettersson scores in severe haemophilia. Acta Radiol. 2002;43(5):528-32.

25. Earnshaw SR, Graham CN, McDade CL, Spears JB, Kessler CM. Factor VIII alloantibody inhibitors: cost analysis of immune tolerance induction vs. prophylaxis and on-demand with bypass treatment. Haemophilia. 2015;21(3):310-19.

26. Arias E. United States life tables, 2011. Natl Vital Stat Rep. 2015;64(11):1-63. 27. Plug I, Van Der Bom JG, Peters M, et al. Mortality and causes of death in patients with hemophilia, 1992-2001: a prospective cohort study. J Thromb Haemost. 2006;4(3):510-16.

28. Farrugia A, Cassar J, Kimber MC, et al. Treatment for life for severe haemophilia A: a cost-utility model for prophylaxis vs. on-demand treatment. Haemophilia. 2013;19(4):e228-38. 
29. Advate (antihemophilic factor [recombinant], plasma/albumin-free method). Baxter Healthcare. 2011. Available at: https://www.fda.gov/ media/70008/download. Accessed May 11, 2020.

30. Hay CR, DiMichele DM; International Immune Tolerance Study. The principal results of the International Immune Tolerance Study: a randomized dose comparison. Blood. 2012;119(6):1335-44.

31. IBM. Micromedex RED BOOK [Database]. Available at: https://www.ibm. com/products/micromedex-red-book. Accessed May 11, 2020.

32. Fryar CD, Gu Q, Ogden CL. Anthropometric reference data for children and adults: United States, 2007-2010. Vital Health Stat 11. 2012;(252):1-48. Available at: https://www.cdc.gov/nchs/data/series/sr_1l/srl1_252.pdf. Accessed May 11, 2020.

33. O'Hara J, Walsh S, Camp C, et al. The relationship between target joints and direct resource use in severe haemophilia. Health Econ Rev. 2018;8(1):1.

34. Centers for Medicaid \& Medicare Services. Physician fee schedule search. Updated April 3, 2020. Available at: http://www.cms.gov/apps/physician-fee-schedule/overview.aspx. Accessed May 11, 2020.

35. Kozma CM, Slaton T, Paris A, Edgell ET. Cost and utilization of healthcare services for hip and knee replacement. J Med Econ. 2013;16(7):888-96.

36. Agency for Healthcare Research and Quality. Healthcare Cost and Utilization Project (HCUPnet). Available at: https://hcupnet.ahrq.gov/. Accessed May 11, 2020.

37. Zakieh A, Siddiqui AH. Emergency department utilization by haemophilia patients in United States. Haemophilia. 2017;23(3):e188-e193.

38. Zhou ZY, Koerper MA, Johnson KA, et al. Burden of illness: direct and indirect costs among persons with hemophilia A in the United States. J Med Econ. 2015;18(6):457-65.

39. Fischer K, Bom JG, Mauser-Bunschoten EP, Roosendaal G, Berg HM, Effects of haemophilic arthropathy on health-related quality of life and socio-economic parameters. Haemophilia. 2005;11(1):43-48.

40. Bureau of Labor Statistics. Employer costs for employee compensation. September 2017. Available at: https://www.bls.gov/news.release/archives/ ecec_12152017.pdf. Accessed May 11, 2020.

41. Su J, Zhou J, Buckley B, Rising T, Hou Q, Jain N. The immune tolerance induction factor utilizations and costs for the management of male hemophilia-a patients who developed inhibitors [abstract]. Blood. 2016;128(22):4758. Available at: https://ashpublications.org/blood/ article/128/22/4758/101573/The-Immune-Tolerance-Induction-FactorUtilizations. Accessed May 20, 2020.
42. Institute for Clinical and Economic Review. Emicizumab for hemophilia A with inhibitors: effectiveness and value. Final evidence report. April 16, 2018. Available at: https://icer-review.org/wp-content/uploads/2017/08/ICER_ Hemophilia_Final_Evidence_Report_041618.pdf. Accessed May 11, 2020.

43. Jimenez-Yuste V, Auerswald G, Benson G, et al. Achieving and maintaining an optimal trough level for prophylaxis in haemophilia: the past, the present and the future. Blood Transfus. 2014;12(3):314-19.

44. Osooli M, Lovdahl S, Steen Carlsson K, et al. Comparative burden of arthropathy in mild haemophilia: a register-based study in Sweden. Haemophilia. 2017;23(2):e79-e86.

45. Ling M, Heysen JP, Duncan EM, Rodgers SE, Lloyd JV. High incidence of ankle arthropathy in mild and moderate haemophilia A. Thromb Haemost. 2011;105(2):261-68.

46. Thornburg CD. Physicians' perceptions of adherence to prophylactic clotting factor infusions. Haemophilia. 2008;14(1):25-29.

47. Batsuli G, Zimowski KL, Tickle K, Meeks SL, Sidonio RF Jr. Immune tolerance induction in paediatric patients with haemophilia A and inhibitors receiving emicizumab prophylaxis. Haemophilia. 2019;25(5):789-96.

48. Medical and Scientific Advisory Council. MASAC recommendations concerning products licensed for the treatment of hemophilia and other bleeding disorders. March 16, 2020. Available at: https://www.hemophilia. org/Researchers-Healthcare-Providers/Medical-and-Scientific-AdvisoryCouncil-MASAC/MASAC-Recommendations/MASAC-RecommendationsConcerning-Products-Licensed-for-the-Treatment-of-Hemophilia-and-OtherBleeding-Disorders. Accessed May 11, 2020.

49. Shima M, Nogami K, Nagami S, et al. Every 2 weeks or every 4 weeks subcutaneous injection of emicizumab in pediatric patients with severe hemophilia A without inhibitors: a multi-center, open-label study in Japan (HOHOEMI study). Blood. 2018;132(Suppl 1):1186.

50. Callaghan M, Negrier C, Paz-Priel I, et al. Emicizumab treatment is efficacious and well tolerated long term in persons with haemophilia A (PwHA) with or without FVIII inhibitors: pooled data from four HAVEN studies. Presented at: ISTH Academy Congress; July 9, 2019; Melbourne, Australia.

51. Tarantino MD, Lail A, Donfield SM, et al. Surveillance of infectious complications associated with central venous access devices in children with haemophilia. Haemophilia. 2003;9(5):588-92.

52. Valentino LA, Kawji M, Grygotis M. Venous access in the management of hemophilia. Blood Rev. 2011;25(1):11-15. 


\section{APPENDIX A Summary of Key Model Choices and Assumptions with Rationales}

Model Choice and Assumption

Patients were assumed to adhere to treatment throughout the modeled time horizon, not considering treatment discontinuation.

For persons with severe HA without inhibitors, the ABRs of patients aged $<12$ years were assumed the same as those of patients aged $\geq 12$ years.

The risk of developing FVIII inhibitors was contingent on the cumulative days of exposure to FVIII.

The risk of developing arthropathy was contingent on the number of joint bleeds having experienced.

Patients receiving prophylaxis were assumed to have the same risk of death as patients with mild to moderate HA.

$A B R=$ annual bleed rate; FVIII = factor VIII; $H A=$ hemophilia $A$.
Rationale

Treatment adherence was not considered because of the lack of supporting data. This could be regarded as a conservative assumption, since nonadherence may be more common in the FVIII arm and, therefore, could have larger negative effect on FVIII's clinical benefits. ${ }^{16}$

The ABRs of patients aged $<12$ years were not available from clinical trials. A small Japanese trial of pediatric patients without inhibitors found consistent results. The assumption was supported with clinical expert opinion.

This was supported with literature, that exposure to FVIII increases the risk of developing FVIII inhibitors. $8-11$

This was supported with literature, that 12.6 joint bleeds increase the Pettersson score by 1 , and the Pettersson score of 1 and above indicates arthropathy and of 28 indicates the requirement of surgery. $23-25$

The goal of prophylaxis is to maintain the blood clotting factor level close to patients with mild to moderate HA. This was supported with literature. 27,28 

Aged 1 Year with Severe Hemophilia A Receiving Emicizumab or FVIII Prophylaxis ${ }^{a}$

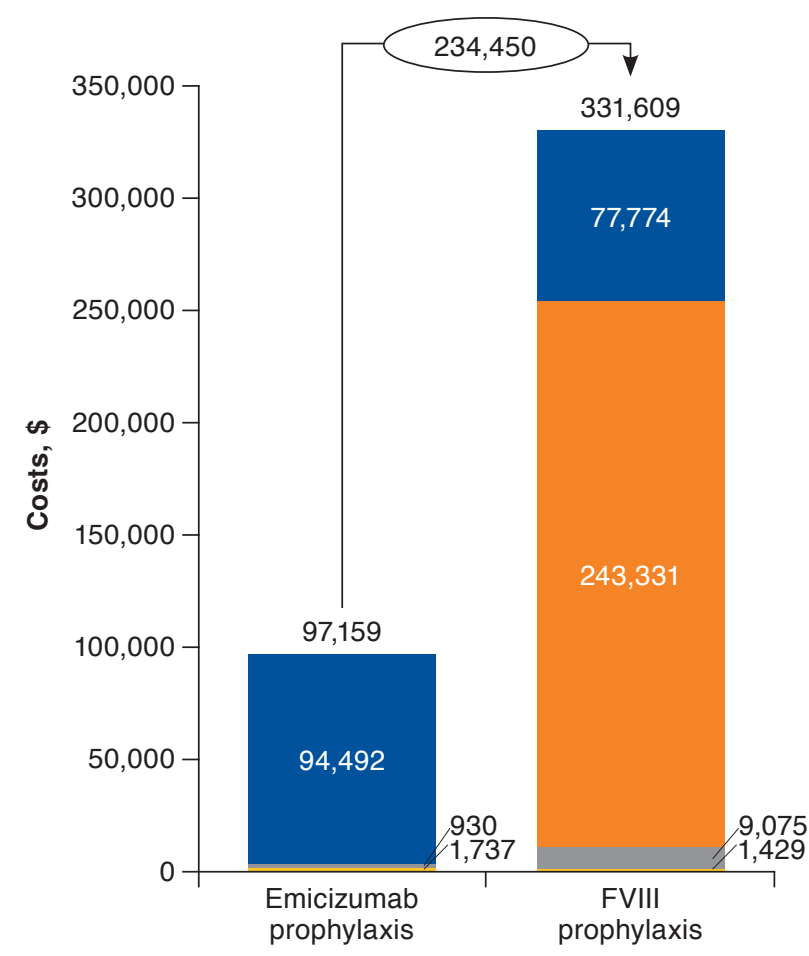

1 year

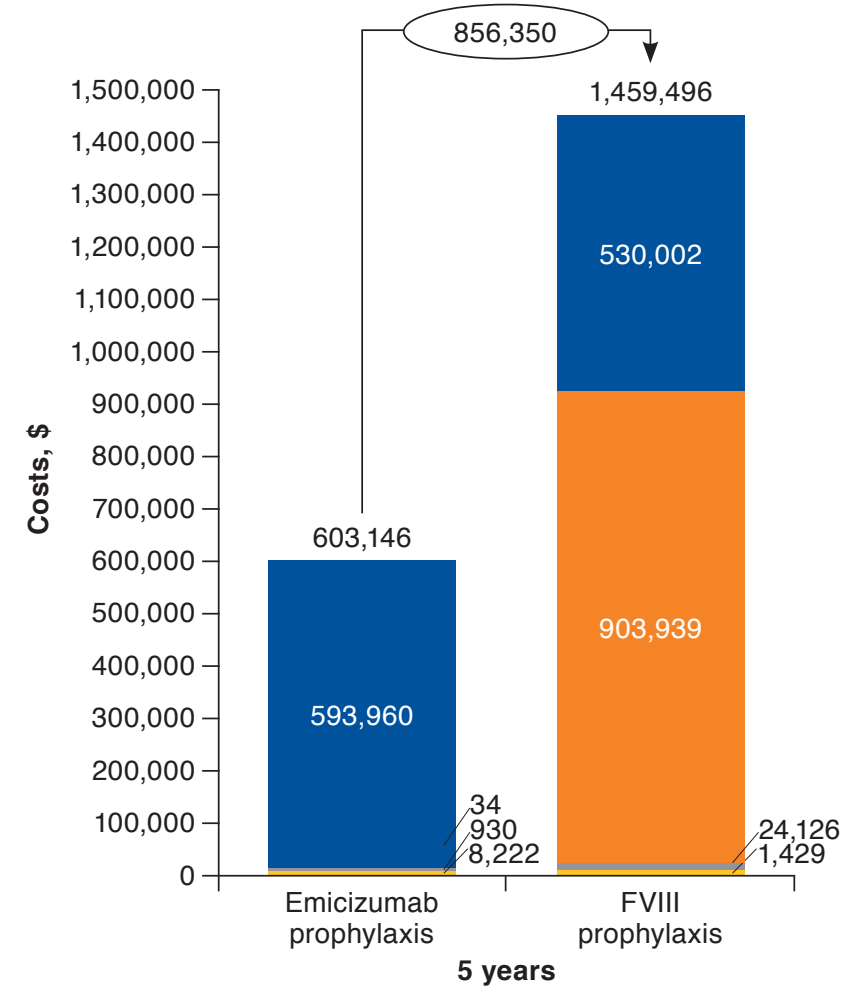

Prophylactic treatment cost

Inhibitor-related treatment cost

Medical cost

Serious adverse event cost

Indirect cost

Note: All costs were inflated to 2018 U.S. dollars.

aThe categories contributing a smaller amount to the total costs included medical costs (\$1,737 for emicizumab and \$9,075 for FVIII over 1 year, and \$8,222 for emicizumab and \$24,126 for FVIII over 5 years); serious adverse event costs (\$930 and \$1,429 for emicizumab and FVIII, respectively, over 1 year and 5 years); and inhibitor-related treatment costs ( $\$ 34$ for emicizumab over 5 years).

FVIII = factor VIII 